\title{
Evidence for triggered star formation in the Carina Flare supershell
}

\author{
Joanne Dawson, A. Kawamura, N. Mizuno, T. Onishi and Y. Fukui \\ Department of Astrophysics, Nagoya University, Chikusa-ku, Nagoya, 464-8602, Japan. \\ Email: joanne@a.phys.nagoya-u.ac.jp
}

\begin{abstract}
Theory predicts the triggered formation of molecular clouds stars through the fragmentation and collapse of swept-up ambient gas. Yet the majority of Galactic HI shells show no more than a scattering of small molecular clouds. The Carina Flare supershell (Fukui et al. 1999 ) is a rare example of an HI shell with a striking molecular component. Here we present the large-scale morphology of the molecular and atomic gas and the location of YSO candidates. A detailed look at two molecular clumps in the shell walls reveals active, intermediate mass star forming regions at various stages of early evolution.
\end{abstract}

Keywords. ISM: bubbles, ISM: structure, stars: formation.

New HI data from the Parkes SGPS reveals the Carina Flare to be a galactic chimney. The molecular and atomic components show good correspondence in position and velocity. Morphological features include molecular clouds with cometary HI tails and starforming molecular clumps in HI filaments. IRAS YSO candidates are scattered across the region and their correlation with loop-like structures of the supershell provides evidence for star formation occurring preferentially in the shell walls. Two star-forming molecular clouds have been examined in detail using ${ }^{12} \mathrm{CO},{ }^{13} \mathrm{CO}$ and $\mathrm{C}^{18} \mathrm{O}(\mathrm{J}=1-0)$ observations made with the NANTEN and SEST telescopes, combined with IR and optical survey data. We find evidence for ongoing intermediate mass star formation in both. We suggest that the Carina Flare is a promising candidate for the triggered formation of both stars and their parent molecular clouds.

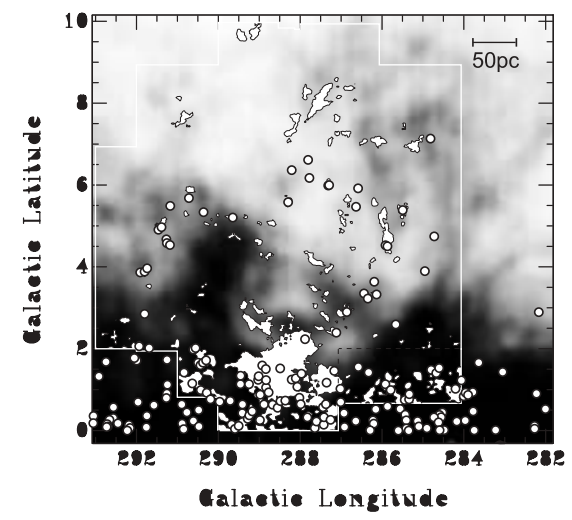

Figure 1. Parkes SGPS HI $21 \mathrm{~cm}$ (greyscale), NANTEN ${ }^{12} \mathrm{CO}(\mathrm{J}=1-0)$ (contours) \& IRAS YSO candidates (circles) selected from the point source catalogue. All emission is integrated over the velocity range $-35<\mathrm{v}_{l s r}<-3 \mathrm{kms}^{-1}$. The white box marks the region observed in ${ }^{12} \mathrm{CO}(\mathrm{J}=1-0)$. Carina Flare basic parameters: distance $\sim 2.6 \pm 0.4 \mathrm{kpc}$; expansion velocity $\sim 8 \mathrm{kms}^{-1}$; size $\sim 250 \times 350 \mathrm{pc}$; atomic mass $\sim 2 \times 10^{5} \mathrm{M}_{\odot} ;$ molecular mass $\sim[1-3] \times 10^{5} \mathrm{M}_{\odot}$.

\section{Reference}

Fukui, Y., Onishi, T., Kawamura, A., Tachihara, K., Yamaguchi, R., Mizuno, A., \& Ogawa, H. 1999, PASJ 751, 51 